\title{
A High Current Efficiency CMOS LDO Regulator with Low Power Consumption and Small Output Voltage Variation
}

\author{
Behnam Samadpoor Rikan*, Hamed Abbasizadeh*, Ji-Hun Kang*, \\ Kang-Yoon Lee L $^{* \star}$
}

\begin{abstract}
In this paper we present an LDO based on an error amplifier. The designed error amplifier has a gain of $89.93 \mathrm{~dB}$ at low frequencies. This amplifier's Bandwidth is $50.8 \mathrm{MHz}$ and its phase margin is $59.2^{\circ}$. Also we proposed a BGR. This BGR has a low output variation with temperature and its PSRR at $1 \mathrm{KHz}$ is $-71.5 \mathrm{~dB}$. For a temperature variation from $-40^{\circ} \mathrm{C}$ to $125^{\circ} \mathrm{C}$ we have just $9.4 \mathrm{mV}$ variation in $3.3 \mathrm{~V}$ LDO output. Also it is stable for a wide range of output load currents $[0-200 \mathrm{~mA}]$ and a $1 \mu \mathrm{F}$ output capacitor and its line regulation and especially load regulation is very small comparing other papers. The PSRR of proposed LDO is $-61.16 \mathrm{~dB}$ at $1 \mathrm{KHz}$. Also we designed it for several output voltages by using a ladder of resistors, transmission gates and a decoder. Low power consumption is the other superiority of this LDO which is just $1.55 \mathrm{~mW}$ in full load. The circuit was designed in $0.35 \mu \mathrm{m}$ CMOS process.
\end{abstract}

Key words: Low-dropout(LDO) Regulator, Bandgap Reference(BGR), Error amplifier, Load Regulation, Line Regulation

\section{Introduction}

These days power management is an important consideration in portable electronic devices such as cellular phones, hand-held computers, and particularly implanted wireless biomedical chips.

* College of Information and Communication Engineering, Sungkyunkwan University

$\star$ Corresponding author

klee@skku.edu, 031-299-4954

※ Acknowledgment

"This research was supported by the MSIP(Ministry of Science, ICT\&Future Planning), Korea, under the ITRC(Information Technology Research Center)"

Support Program : "This work was supported by IC Design Education Center(IDEC)"

Manuscript received Jan. 15, 2014; revised Feb. 25,2014 ; accepted Feb, 25. 2014
The purpose of power management is to reduce power consumption and increase the battery life. Low-dropout(LDO) regulators are widely used alone in many integrated power management circuits but are also used as post regulators of switching converters, as they can provide wideband regulated low noise supply voltage for noise-sensitive analog and RF loads. Traditional linear LDO regulators usually consist of a two-stage error amplifier, a power output stage and a negative feedback loop. The error amplifier detects the error between the reference and the output voltage. There are several performance metrics to be fulfilled when designing an LDO, such as closed-loop stability for a wide range of loads, static regulation in front of input voltage and load variations, and fast rejection of steep transient load variations [1, 2]. 
Operational amplifiers (OP-AMPS) have become one of the core components in analog and mixed signal (AMS) design. The proposed error amplifier here is a two stage high speed CMOS op-amp. The design procedure involves designing the bias circuit, the differential input pair, and gain stage [3].

As we mentioned the error amplifier detects the error between the reference and the output voltage. The reference voltage is produced by a Bandgap Reference(BGR). Precision BGRs are critical building blocks for a variety of analog and mixed signal electronic devices such as data converters, PWM controllers, oscillators, operational amplifiers, linear regulators and PLLs. Undoubtedly the reference voltage accuracy plays a significant part in determining the performance of all subsequent circuits, which depend on an accurate and stable reference $[4,5]$.

All these parts gathered together and make an LDO. Conventional LDOs just give an output which's variation with temperature is low. Unfortunately most of the designed LDO's can't provide all of the LDO performance metrics together and each of them has a low performance in one aspect. And also their load current variation is in small range and they can't suffer high load currents. In this paper we propose an LDO with high stability for line, load and temperature variations. The load current has been increased comparing other papers and also this LDO can provide several needed voltages at the output.

In section II of this paper we present the proposed BGR. Section III describes the proposed error amplifier and its characteristics. Then in section IV we will see the structure of LDO. Finally the simulation results will be shown in section $\mathrm{V}$.

\section{Bandgap Reference}

To generate a stable voltage against process and temperature variations, a BGR circuit is adopted. BGR circuit was first proposed by Widlar [6] and was further developed by Brokaw [7], that are most commonly used for all analog circuits for providing reference voltages. We will use it in our LDO [1,8]. The conventional BGR structure is shown in Fig.1. which is composed of CMOS opamp, BJTs and resistors.

The relation between the diode current and $\mathrm{V}_{\mathrm{BE}}$ voltage can expressed as:

$$
I=I_{s} \times\left(e^{\frac{V_{B E}}{V_{T}}}-1\right)
$$

or

$$
V_{B E}=V_{T} \times \ln \frac{I}{I_{S}}
$$

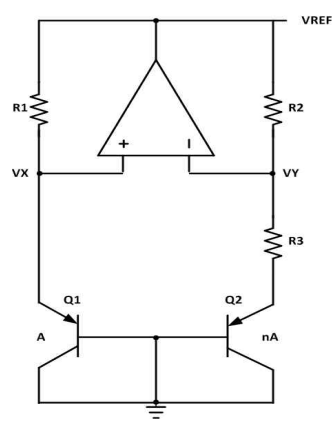

Fig. 1. Conventional BGR circuit

Here $\quad V_{T}=K T / q$, and $\quad K=1.38 \times 10^{-23} \mathrm{~J} / K \quad$ is Boltzmann's constant and $q=1.6 \times 10^{-19} \mathrm{C}$ is electronic charge. $\triangle V_{B E}$ is the forward voltage difference between Q1 and Q2 transistors for different current densities.

$$
\triangle V_{B E}=V_{T} \times \ln (n)
$$

In conventional $\mathrm{BGR}$ circuit, $V_{X}$ and $V_{Y}$ nodes are controlled by opamp circuit and BGR output voltage $\mathrm{V}_{\mathrm{REF}}$ is given by

$$
V_{R E F}=V_{B E}+V_{T} \times \ln (n)\left(1+R_{2} / R_{3}\right)
$$

The BGR here uses the same structure as conventional BGRs (Fig.2.). Transistors M1-M8 are op-amp transistors. A start up circuit (M11-M14) also added to ensure BGR's start up situation. Proportional to absolute temperature (PTAT) current is created through M9 and M10 and temperature independent voltage is created 
by using $\mathrm{R} 4$ in $\mathrm{V}_{\mathrm{REF}}$. To overcome the supply dependence caused by channel-length modulation of MOS devices, we have used cascade topology for both NMOS and PMOS transistors. Also $R_{2}$ and $R_{3}$ sustain proper voltages to allow MOSFETs to remain in saturation [8].

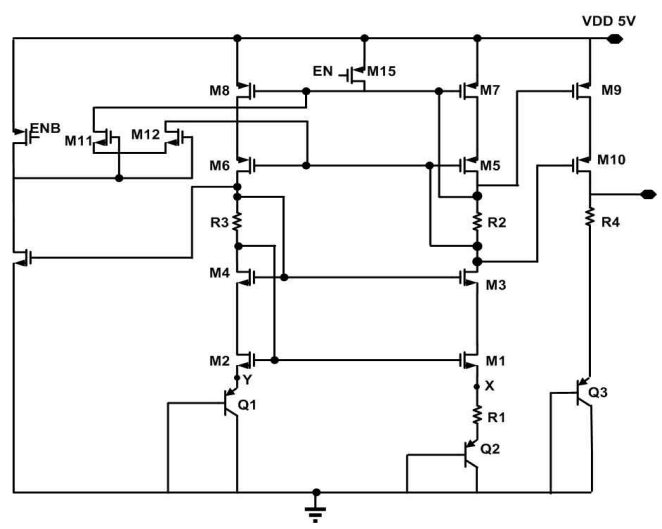

Fig. 2. BGR proposed to use as $\mathrm{V}_{\mathrm{REF}}$ in $\mathrm{LDO}$

This BGR produces $1.2 \mathrm{~V}$ for the error amplifier of LDO. It has a very small variation for temperature sweep of $-40^{\circ} \mathrm{C}$ to $125^{\circ} \mathrm{C}$ which is important to be used as reference voltage of LDO. Also high PSRR is the other priority of this BGR which is so important parameter in these days BGR and LDO design. Tablel shows this BGR's characteristics.

Table 1. BGR circuits performance parameters

\begin{tabular}{|c|c|c|}
\hline & This Work & {$[4]$} \\
\hline $\begin{array}{c}\text { Reference Output } \\
\text { Voltage }\end{array}$ & $1.202 \mathrm{~V}$ & $1.23 \mathrm{~V}$ \\
\hline $\begin{array}{c}\text { Voltage Variation } \\
\text { for }-40^{\circ} \mathrm{C} \text { to } 125^{\circ} \mathrm{C}\end{array}$ & $\pm 0.1325 \%$ & $\pm 0.1 \%$ \\
\hline PSRR & $\begin{array}{c}-72.5 \mathrm{~dB} @ \\
100 \mathrm{~Hz} \\
-71.5 \mathrm{~dB} @ \\
1 \mathrm{KHz}\end{array}$ & $\begin{array}{c}-31 \mathrm{~dB} \\
\text { C } 10 \mathrm{~Hz}\end{array}$ \\
\hline Current & $42.8 \mathrm{uA}$ & $180 \mathrm{uA}$ \\
\hline
\end{tabular}

\section{Error Amplifier}

The op-amp design at schematic level is mainly divided into three main parts, the biasing circuit, the input differential pair and gain stage. In this paper we propose a two stage op-amp with the bias circuit and a load capacitor $\mathrm{C}_{\mathrm{L}}$ as shown in Fig.3. [3].

For a good performance of LDO this amplifier should have high gain and proper phase margin. Gain of this op-amp is

$$
A_{V}=A_{V 1} \times A_{V 2}
$$

$A_{v 1}$ is the first stage or differential input stage's gain. The transistor M9 and M10 are the p-channel input pair and the M11 and M12 are the $n$-channel active load current mirrors. M13 and M14 are the biasing transistors. The differential pair is active loaded with the NMOS current mirror.

$$
R_{O}=r_{d s 10} \| r_{d s 12}
$$

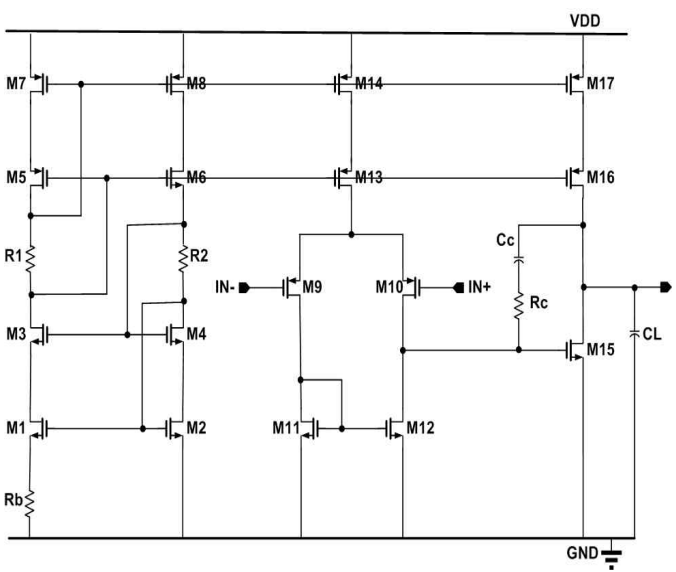

Fig. 3. two stage op-amp with load capacitor $\mathrm{C}_{\mathrm{L}}$

So the differential gain of the differential pair is given by

$$
A_{V 1}=g_{m 9}\left(r_{d s 10} \| r_{d s 12}\right)
$$

Also we have gain stage, which is the second stage of two stage op-amp. Gain stage, is an $\mathrm{n}$-channel common source amplifier, active loaded 
with the PMOS current source. The gain of this stage is

$$
A_{V 2}=-g_{m 15}\left(r_{d s 16} \| r_{d s 15}\right)
$$

A miller compensation capacitor is added between the input and the output of the gain stage so as to move the pole lowest in frequencies to lower frequencies and the one at high frequency to higher frequencies and achieve more stability at the cost of reduced speed. To get required GBW $[9,10]$ are used

$$
f_{u n}=\frac{g_{m 10}}{2 \Pi C_{c}}
$$

Where $f_{u n}$ is the GBW and $C_{c}$ is the miller compensation capacitor and controls the dominant first pole.

Resistor $R_{c}$ is connected in series with $C_{c}$ to cancel the right half plane zero altogether[10],

$$
R_{c}=\frac{1}{g_{m 7}}
$$

Also the value of $R_{c}$ is increased to move the zero further into left half plane.

Table2 shows this amplifiers performance parameters.

Table 2. Results for proposed error op-amp

\begin{tabular}{|c|c|c|}
\hline & This work & {$[3]$} \\
\hline Open Loop Gain & $89.93 \mathrm{~dB}$ & $>90 \mathrm{~dB}$ \\
\hline Bandwidth & $50.77 \mathrm{MHz}$ & $57.01 \mathrm{MHz}$ \\
\hline Phase Margin & $59.2^{\circ}$ & $56^{\circ}$ \\
\hline $\mathrm{C}_{\mathrm{c}}$ & $800 \mathrm{fF}$ & $800 \mathrm{fF}$ \\
\hline $\mathrm{R}_{\mathrm{c}}$ & $1.2 \mathrm{~K} \mathrm{Ohm}$ & $8 \mathrm{~K} \mathrm{ohm}$ \\
\hline $\mathrm{C}_{\mathrm{L}}$ & $100 \mathrm{fF}$ & $10 \mathrm{pF}$ \\
\hline
\end{tabular}

\section{Low Dropout Regulator}

Block diagram of proposed LDO is shown in Fig.4. It is composed of a BGR, an error amplifier, a power MOS (MP) and a voltage divider composed of $\mathrm{R} 1$ and $\mathrm{R} 2$. More than $200 \mathrm{~mA} \mathrm{I} \mathrm{I}_{\text {load }}$ and $1 \mathrm{uF}$ capacitor is used for full load situation in our simulations.

As we mentioned, $\mathrm{Mp}$ is a power transistor so its aspect ratio is larger than other transistors to provide $200 \mathrm{~mA}$ load current [11].

To get several fixed output voltages (not only 3.3V output voltage) as shown in Fig.4. we replaced $\mathrm{R} 1$ with a ladder of 8 resistors, so according to voltages of Table3, we can get the needed output from the related node. To control the output voltage we use 8 transmission gates and control them with a $3 \times 8$ decoder.

Figure of Merit (FOM) can be calculated as below

$$
F O M=\frac{T_{R} \times I_{Q}}{I_{O U T(M A X)}}
$$

where

$$
T_{R}=\frac{C_{O U T} \times \Delta V_{O U T}}{I_{O U T(M A X)}}
$$

And $\mathrm{I}_{\mathrm{Q}}$ is the current passing through $\mathrm{R} 2[11,12]$.

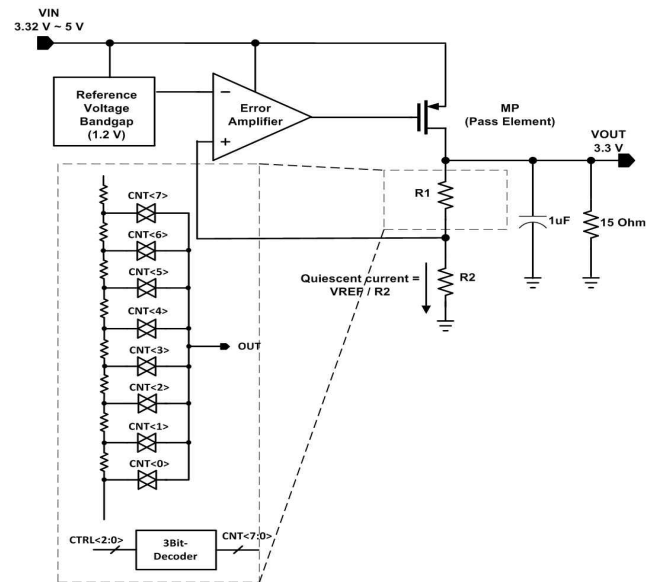

Fig. 4. Block diagram of proposed LDO 
Table 3. Output voltages control bits

\begin{tabular}{|c|c|}
\hline $\begin{array}{c}\text { Control bit } \\
<2: 0>\end{array}$ & V out(V) \\
\hline 000 & 3.61 \\
\hline 001 & 3.53 \\
\hline 010 & 3.45 \\
\hline 011 & 3.38 \\
\hline 100 & 3.31 \\
\hline 101 & 3.24 \\
\hline 110 & 3.18 \\
\hline 111 & 3.12 \\
\hline
\end{tabular}

\section{Simulation Results}

The proposed LDO topology has been designed and simulated in $0.35 \mu \mathrm{m}$ CMOS process. The simulations have been done in full load and for other load situations we will even have better results. These results are for $3.3 \mathrm{~V}$ output voltage with $220 \mathrm{~mA}$ load current and $1 \mu \mathrm{F}$ output capacitor and the performance for all of the other output voltages in Table 3 will be the same.

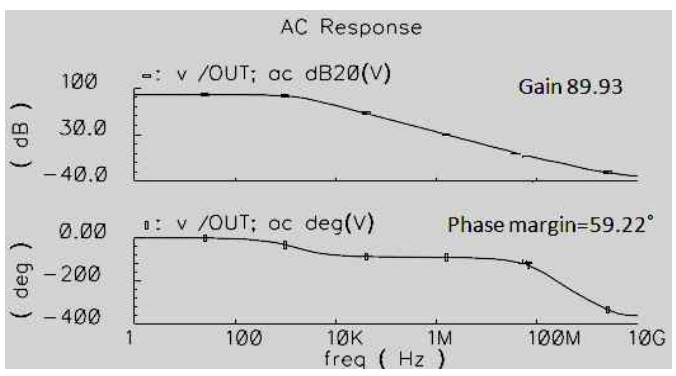

Fig. 5. Error amplifier's gain and PM

First of all we can see the error amplifier gain and phase margin in Fig.5. According to the simulations, we got to the gain of almost $90 \mathrm{~dB}$ and $59.2^{\circ}$ phase margin and $50.77 \mathrm{MHz}$ bandwidth. These results shows this error amplifier can be used in LDO and it will have proper performance.

Line regulation and load regulation of proposed LDO are shown in Fig.6. and Fig.7. respectively.

As we can see in Fig.6. the line regulation for this LDO is $1.937 \mathrm{mV}$ (for $4.7<\mathrm{VDD}<5.7$ ).

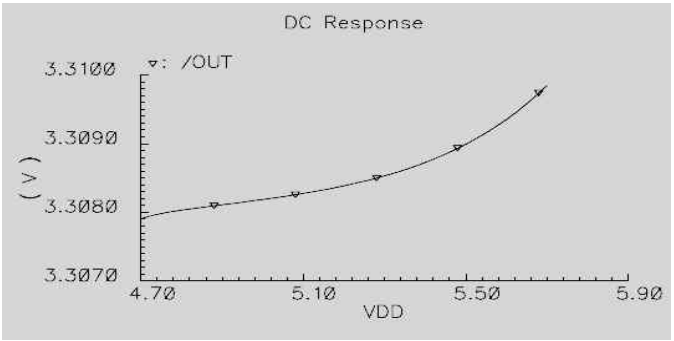

Fig. 6. Line Regulation

This means that if our VDD have $1 \mathrm{~V}$ variation the output voltage will vary less than $2 \mathrm{mV}$. This shows LDO is stable for supply voltage variations and it is important issue in LDO design.

Also load regulation of this LDO is $174.334 \mu \mathrm{V}$ for $0<\mathrm{I}_{\text {LOAD }}<200 \mathrm{~mA}$, which is considerably low, comparing other works. It means we can get a large range of current in the output without so much changes in the performance and so LDO is stable for this range of load current variation. This is one of the priority of this work which can suffer comparable large ranges of load currents comparing other works.

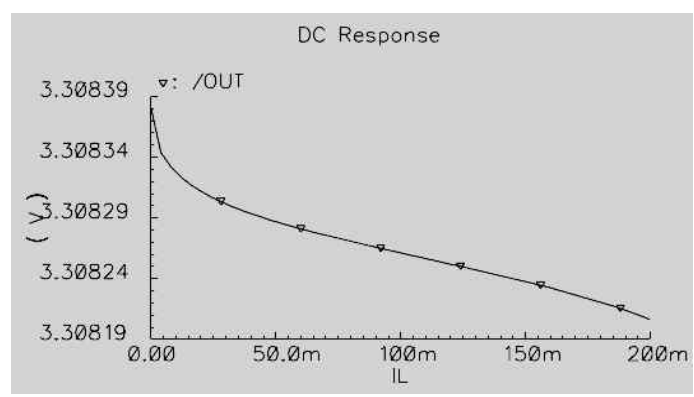

Fig. 7. Load Regulation

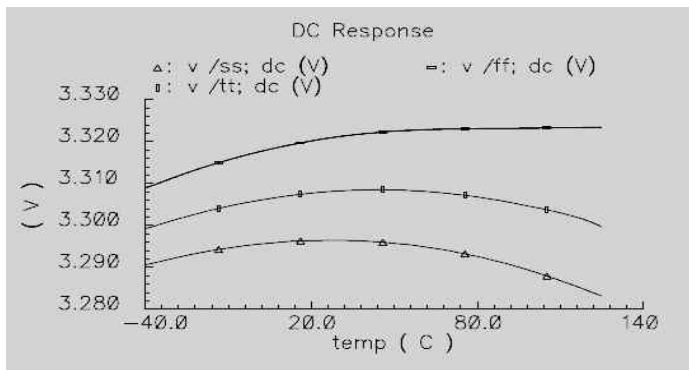

Fig. 8. Output voltage variation of LDO with temperature variation in different corners 
Fig.8. shows output voltage variation with temperature. As we can see, for $-40^{\circ} \mathrm{C}$ to $125^{\circ} \mathrm{C}$ temperature variation, we have just $9.4 \mathrm{mV}$ output voltage variation in Typical state. It shows the stability of the circuit to the temperature variation.

In Fig.9. we can see the 8 different outputs of the LDO. These outputs are stable for temperature variations and line and load regulations as $3.3 \mathrm{~V}$ output node does. These 8 voltages can be obtained using a resistor ladder, transmission gates and $3 \times 8$ decoder.

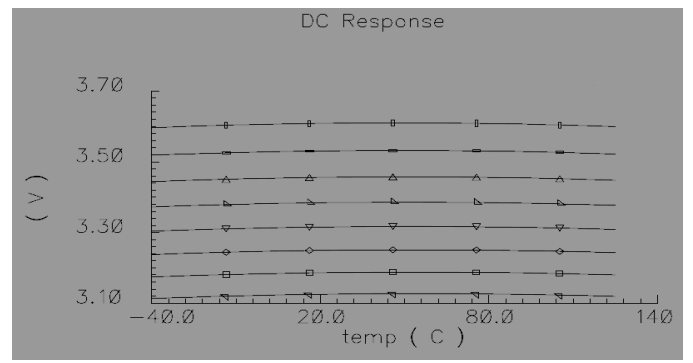

Fig. 9. Different output variations of LDO with temperature variations

In Fig.10. the output voltage variation of the BGR for temperature variation has been shown. We can see the variation is small in all 3 shown corners. It is important for BGR to be stable for temperature variations which we can see in our design

Dropout voltage of the LDO for output of $3.3 \mathrm{~V}$ is $3.32 \mathrm{~V}$ as shown in Fig.11. It means that for VDD voltages over $3.32 \mathrm{~V}$ we can get the output voltage of $3.3 \mathrm{~V}$.

Fig.12. shows PSRR of proposed BGR and LDO respectively. High PSRR is very important issue for the good performance of the LDO. As we can see until $1 \mathrm{KHz}$ we have almost -61dB PSRR which shows high performance of this LDO.

Current consumption of BGR and LDO are $42.68 \mu \mathrm{A}$ and $267.14 \mu \mathrm{A}$ respectively, so power consumption of this LDO is $1.55 \mathrm{~mW}$.

Settling time of circuit is $141 \mu \mathrm{s}$ and FOM is $0.33 \mathrm{nS}$. Table4 presents performance summary. We should emphasize again that load current of this LDO can be even more than $200 \mathrm{~mA}$. It has high PSRR and low power consumption. Also it is stable for line, load and temperature variations in high performance Fig.13. shows the layout of the proposed LDO. The total area is $0.1 \mathrm{~mm}^{2}$ and the post simulation results confirm our simulation results.

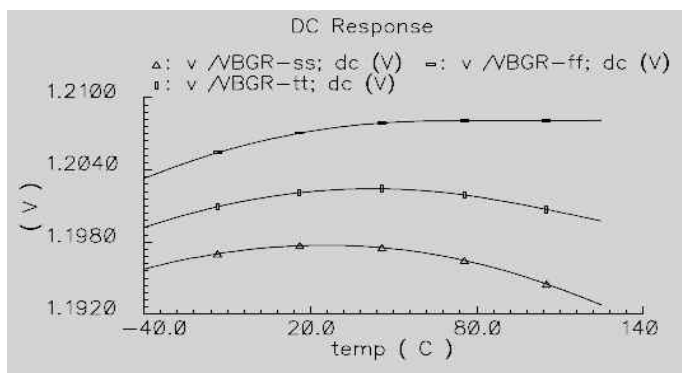

Fig. 10. Output Voltage variation of BGR with temperature variation in different corners

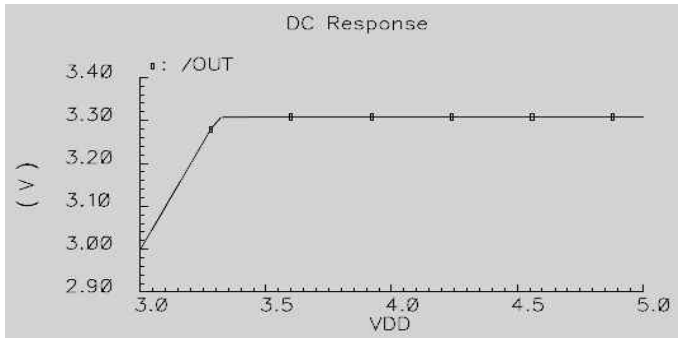

Fig. 11. Dropout voltage of LDO

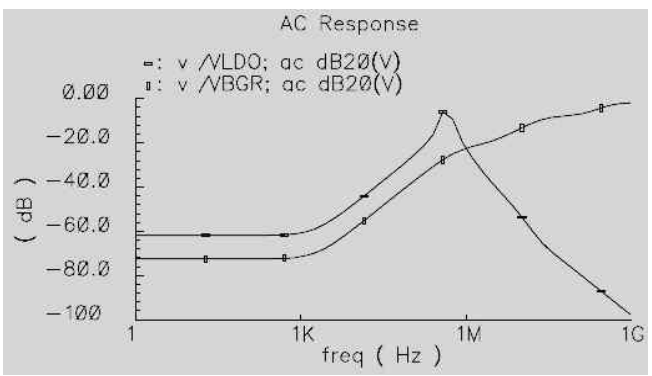

Fig. 12. PSRR of proposed LDO and BGR

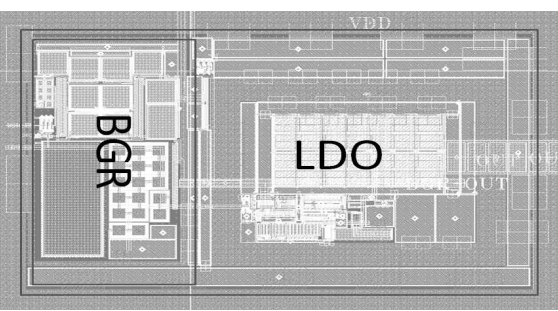

Fig. 13. Layout of LDO and BGR 
Table 4. Performance summary and comparison with recently reported LDO simulation results

\begin{tabular}{|c|c|c|c|c|c|c|c|c|}
\hline & {$[13]$} & {$[14]$} & {$[15]$} & {$[16]$} & {$[12]$} & {$[17]$} & {$[2]$} & This Work \\
\hline Technology[um] & 0.5 & 0.35 & 0.09 & 0.35 & 0.35 & 0.25 & 0.35 & 0.35 \\
\hline $\mathrm{I}_{\text {load }[\mathrm{mA}]}$ & 160 & 200 & 100 & 50 & 50 & 50 & 100 & 220 \\
\hline $\mathrm{V}_{\text {in }}[\mathrm{V}]$ & 3.3 & $2-5.5$ & 1.2 & $1.2-1.5$ & $1.05-3.5$ & $2-2.5$ & $3-5$ & $3.32-5$ \\
\hline $\mathrm{V}_{\text {out }}[\mathrm{V}]$ & 2.8 & 1.8 & 0.9 & 1 & 0.9 & $1.5-1.97$ & 2.8 & 3.3 \\
\hline $\begin{array}{c}\text { Compensation } \\
\text { Cap.[pF] }\end{array}$ & 5 & 10 & $\times$ & $\times$ & $\times$ & N.A. & $\times$ & 1 \\
\hline Output Cap.[uF] & 2.2 & 1 & 0.0006 & 1 & 1 & 0.05 & 1 & 1 \\
\hline Iq[mA] & 25 & $20-320$ & 6000 & 95 & $4.05-164$ & 100 & $59-189$ & 120 \\
\hline PSRR[dB] & N.A. & $45 @ 20 \mathrm{KHz}$ & N.A. & N.A. & 50 & $45 @ 30 \mathrm{KHz}$ & 56 & $>50 @ 10 \mathrm{KHz}$ \\
\hline Load Reg.[mV/mA] & 1.25 & 0.17 & N.A. & 0.28 & N.A. & 0.08 & 0.025 & $<0.01$ \\
\hline Line Reg.[mV/V] & N.A. & 2 & N.A. & 18 & 1.061 & N.A. & 13.5 & 1.93 \\
\hline Current efficiency[\%] & N.A. & 99.8 & 94.3 & 99.8 & 99.67 & 99.8 & 99.8 & 99.85 \\
\hline$\triangle V_{\text {out }}[\mathrm{mV}]$ & 200 & 54 & 90 & 30 & 6.6 & 15 & 3 & 137 \\
\hline $\mathrm{T}_{\mathrm{R}}[\mathrm{us}]$ & 2.75 & 0.27 & 0.0054 & 0.6 & 0.132 & 0.03 & 0.03 & 0.62 \\
\hline
\end{tabular}

\section{Conclusion}

An LDO based on an error amplifier is presented in this paper. With a ladder of resistors and a $3 \times 8$ decoder, we get 8 different output voltages. The circuit is stable for a wide range of load currents [0-200mA]. Also for source voltage and temperature variations the circuit shows high stability. For temperature variations, the maximum output voltage variation is $9.38 \mathrm{mV}$. PSRR of this LDO in DC is $-62.3 \mathrm{~dB}$ and in $1 \mathrm{KHz}$ is $-61.2 \mathrm{~dB}$ which shows high performance. The power consumption is $1.55 \mathrm{~mW}$.

\section{References}

[1] C. Wang, R. Kuo, T. Tsai, A high precision dropout regulator with nested feedback loops, Microelectronics Journal $42 \quad$ (2011) 966-971.

[2] A. Saberkari, E. Alarcon, Sh.B. Shokouhi, Fast transient current-steering CMOS LDO regulator based on current feedback amplifier. Integer.VLSI J.46 (2013) 165-171.

[3] Y.M. Qureshi, Design and Layout of Two Stage High Bandwith Operational Amplifier, World Academy of Science, Engineering and Technology 712012.

[4] X. Ming, Y. Ma, Z. Zhou, B. Zhang, A High-Precision Compensated CMOS Bandgap Voltage Reference Without Resistors, IEEE
Trans. Circuits Systs-II, VOL. 57, NO. 10, OCTOBER 2010.

[5] M. Andreou, S. Koudounas, J. Georgiou, A Novel Wide-Temperature-Range, $3.9 \mathrm{ppm} /{ }^{\circ} \mathrm{C}$ CMOS Bandgap Reference Circuit, IEEE J. Solide-State Circuits, VOL. 47, NO. 2, FEBRUARY 2012.

[6] R. J. Widlar, "Developments in IC voltage regulators," IEEE J. Solid-State Circuits, vol. SC- 6, pp. 2-7, Feb. 1971.

[7] P. Brokaw, "A simple three terminal IC bandgap reference," IEEE J.Solid- State Circuits, vol. SC-9, pp. 388 - 393, Dec. 1974.

[8] M. K. Adimulam, K. K. Movva, A Low Power CMOS Current Mode Bandgap Reference Circuit with Low Temperature Coefficient of Output Voltage, PRIMEASIA (2012) 144-149.

[9] D. Johns, K. Martin, Analog Integrated Circuit Design. Chichester: Wiley, 1997, pp. 225 227.

[10] D. Johns, K. Martin, Analog Integrated Circuit Design. Chichester: Wiley, 1997, pp. $240-244$.

[11] R. Fathipour, A. Saberkari, H. Martinez, E. Alarcon, High slew rate current mode transconductance error amplifier for low quiescent current output-capacitor less CMOS LDO regulator, Integr.VLSI J. 2013.

[12] Y. Hei, W. H. Ki, A $0.9 \mathrm{~V} 0.35 \mu \mathrm{m}$ adaptively biased CMOS LDO regulator with fast transient response, in: Proceedings of the IEEE International Solid-State Circuits 
Conference (ISSCC'08), 2008, pp. $442-443$.

[13] C.K. Chava, J. Silva-Martinez, A frequency compensation scheme for LDO voltage regulators, IEEE Transactions on Circuits and Systems I 51 (6) (2004) 1041 - 1050.

[14] M. Al-Shyoukh, H. Lee, R. Perez, A transient-enhanced low-quiescent current low-dropout regulator with buffer impedance attenuation, IEEE Journal of Solid-State Circuits 42 (8) (2007) $1732-1742$.

[15] P. Hazucha, T. Karnik, B. Bloechel, C. Parsons, D. Finan, S. Borkar, Area- efficient linear regulator with ultra-fast load regulation, IEEE Journal of Solid- State Circuits 40 (4) (2005) 933 - 940.

[16] T.Y. Man, K.L. Leung, C.Y. Leung, P.K.T. Mok, M. Chan, Development of singletransistor-control LDO based on flipped voltage follower for SoC, IEEE Transactions on Circuits and Systems I 55 (5) (2008) $1392-1401$.

[17] W. Oh, B. Bakkaloglu, A CMOS low-dropout regulator with current-mode feedback buffer amplifier, IEEE Transactions on Circuits and Systems II 54 (10) (2007) 922 - 926.

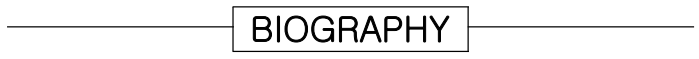

Behnam Samadpoor Rikan (Student Member)

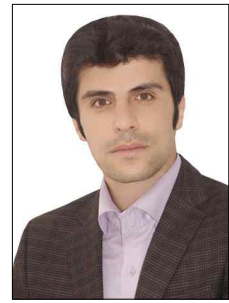
2008 : BS degree in Electrical Engineering, Urmia University. 2013 : MS degree in Electronic Engineering, Qazvin Azad University. 2013 2014 : Ph.D student in College of Information and Communication Engineering, Sungkyunkwan University.
Hamed Abbasizadeh (Student Member)

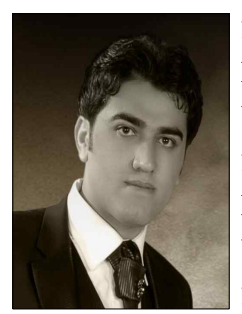
2008: BS degree in Electrical Engineering, Booshehr Azad University. 2012 : MS degree in Electronic Engineering, Qazvin Azad University. 2013 2014: Ph.D student in College of Information and Communication Engineering, Sungkyunkwan University.

Ji-Hun Kang (Student Member)

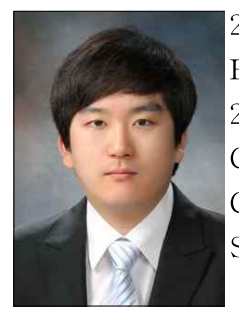
2013 : BS degree in Electronic Engineering, Myoung Ji University. 2013 2014 : Master degree in College of Information and Communication Engineering, Sungkyunkwan University.

Kang-Yoon Lee (Member)

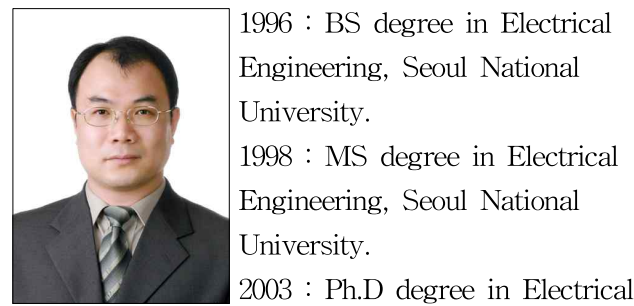

Engineering, Seoul National University 2003 2005 : Research Engineer, GCT Semiconductor Inc.

2005 2011 : Professor, Department of Electronics Engineering, Konkuk University. 2012 2014 : Professor, Department of Information and Communication Engineering, Sungkyunkwan University. 